\section{Early statin therapy beneficial for familial hypercholesterolemia}

Patients with familial hypercholesterolemia have elevated LDL-cholesterol levels from birth, and atherosclerosis can develop at a young age. It is unclear, however, at what age cholesterol-lowering treatment can be initiated safely.

Rodenburg et al. conducted a follow-up study of subjects from an earlier randomized trial. Participants had taken either pravastatin or placebo for the duration of the trial (2 years), and all had been started on pravastatin at the trial's conclusion. At the end of the follow-up study, data were available for 186 children (mean age $13.7 \pm 3.1$ years) who had been taking pravastatin for an average of 4.5 years (range 2.1-7.4 years).

Multivariate analysis showed that the earlier statin treatment was started, the lower carotid intima media thickness (IMT) - a validated surrogate marker for atherosclerotic disease-was at follow-up. According to this model, a $0.003 \mathrm{~mm}$ increase in carotid IMT can be expected for every year that statin therapy is postponed. Along with duration of statin treatment, initial carotid IMT and male sex were also predictors of final IMT.

Early statin therapy seems safe as well as effective-there were no clinical or laboratory adverse events during follow-up and no obvious effects on pubertal development. Longerterm observation is required to confirm these findings and establish the optimum age for the initiation of statin therapy.

Original article Rodenburg J et al. (2007) Statin treatment in children with familial hypercholesterolemia: the younger, the better. Circulation 116: 664-668

\section{Interleukin 1 receptor family member ST2 has prognostic value in dyspnea}

Evidence is accumulating to indicate that measurement of levels of the interleukin 1 receptor family member ST2 might have prognostic value in patients with heart failure (HF). In vitro studies have shown marked parallels between the expression profiles of ST2 and those of $\mathrm{N}$-terminal pro-brain natriuretic peptide, and elevated levels of ST2 have been documented in patients with $\mathrm{HF}$ in small studies.
By use of data from the PRIDE study, Januzzi et al. examined the prognostic utility of ST2 measurement in a group of 593 dyspneic patients including both those with and those without acute destabilized HF. Median ST2 concentrations were significantly higher in patients with $\mathrm{HF}$ than in patients without $(0.50 \mathrm{ng} / \mathrm{ml}$ vs $0.15 \mathrm{ng} / \mathrm{ml} ; P<0.001)$, confirming a possible biological link between ST2 and the presence and severity of HF.

After 1 year of follow-up, 93 patients had died. Median ST2 concentrations were significantly higher among decedents than among survivors $(1.03 \mathrm{ng} / \mathrm{ml}$ vs $0.18 \mathrm{ng} / \mathrm{ml} ; P<0.001)$, and an ST2 concentration of $\geq 0.20 \mathrm{ng} / \mathrm{ml}$ dramatically increased the risk of death at 1 year when the population was considered as a whole (hazard ratio 5.6, 95\% Cl 2.2-14.2), and especially in individuals with HF (hazard ratio $9.3,95 \% \mathrm{Cl} 1.3-17.8)$. Further analyses showed that models incorporating both ST2 and $\mathrm{N}$-terminal pro-brain natriuretic peptide were uniquely able to identify those patients at the highest risk of death. The authors conclude that ST2 is a novel biomarker in HF, and that it is strongly predictive of mortality at 1 year among patients with dyspnea, suggesting that ST2 testing might enable enhanced risk stratification of dyspneic patients.

\footnotetext{
Original article Januzzi JL Jr et al. (2007) Measuremen of the interleukin family member ST2 in patients with acute dyspnea: results from the PRIDE (Pro-Brain Natriuretic Peptide Investigation of Dyspnea in the Emergency Department) study. J Am Coll Cardiol 50: $607-613$
}

\section{Interactions between anti- thrombotic drugs increase the risk of gastrointestinal bleeding}

Co-prescription of antithrombotic medications can increase the chance of gastrointestinal bleeding. The overall risk associated with antithrombotic drug combinations can exceed that which would be expected from combining the risks of each individual drug, suggesting that interactions between the drugs might affect the risk of bleeding.

Delaney and colleagues identified 4,028 cases of gastrointestinal bleeding and 40,171 matched controls from the UK General Practice Research Database. Eligible cases were at least 18 years old, had been diagnosed between 